\title{
Research on Attitude Algorithm of Aircraft Based on Quaternion
}

\author{
Junru Chen \\ No.500, DongChuan Road, MinHang District, Shanghai, China
}

\begin{abstract}
In this paper, the quaternion method is used to get the accurate aircraft attitude. It is introduced coordinate system and attitude angle establishment, attitude angle acquisition and data fusion algorithm in detail. Experiments show that the algorithm effectively suppresses the gyroscope deviation and improves the accuracy of the attitude angle.
\end{abstract}

Keywords-quaternion; coordinate system; attitude angle

\section{INTRODUCTION}

The attitude of the aircraft is an angle between the real flying object and the reference coordinate system. The method of aircraft attitude calculation is based on gyroscope, accelerometer, magnetometer and other sens or data to obtain the attitude angle. There are two methods: Euler angle method and quaternion method. Euler angle can not do the analysis of the whole attitude because of the existence of "Gimbal Lock"; while the quaternion method is difficult to understand, but the calculation is simple and high efficiency. Therefore, this paper mainly based on the quaternion method, combined with Euler angle and rotation matrix for data compensation to achieve aircraft attitude calculation.

\section{SYSTEM STRUCTURE}

In this paper, STM32F407 works as the master chip, combined with acceleration/gyroscope sensor MPU6050 and magnetometer AK8975 to complete the attitude solution. The sensor communicates with the MCU through the I2C interface, and the MCU is connected with the host computer through the UART interface[1]. System structure is shown in Figure 1:

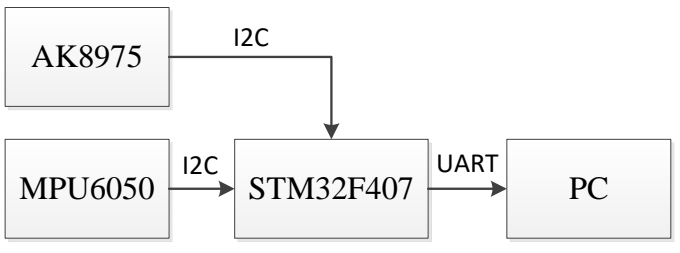

FIGURE I. SYSTEM STRUCTURE

\section{SYSTEM AND ATTITUDE ANGLE}

During the flight, the attitude of plane, missiles and other aircrafts is in change, it is necessary to establish a carrier coordinate system. This paper studies the strap-down inertial navigation system, that is, the gyroscope, the accelerometer and the magnetometer are directly installed on the aircraft, and the attitude matrix is obtained by using the sensor data. So the calculated attitude matrix is the attitude of the carrier coordinate system relative to the reference coordinate system. Therefore, to determine the attitude of the aircraft, we must first calibrate the reference coordinate system and the carrier coordinate system.

\section{A. Definition of Coordinate System}

\section{1) Reference coordinate system $(N)$}

The reference coordinate system is used in the ground remote control command system. It usually adopts the geographical coordinate system $(\mathrm{G})$, selects the north, east and sky (NEU) as the three axis positive direction, that is, its $\mathrm{X}$ axis points to the east, $\mathrm{Y}$ axis points to the north and $\mathrm{Z}$ axis is perpendicular to the ground pointing to the sky.

\section{2) Carrier coordinate system $(B)$}

Its origin is located in the centroid of the carrier. $\mathrm{X}$ axis is along the Horizontal axis of the carrier to the right, $\mathrm{Y}$ axis is along the direction of carrier forward, $\mathrm{Z}$ axis is vertically up the carrier. Carrier coordinate system is shown in Figure 2:

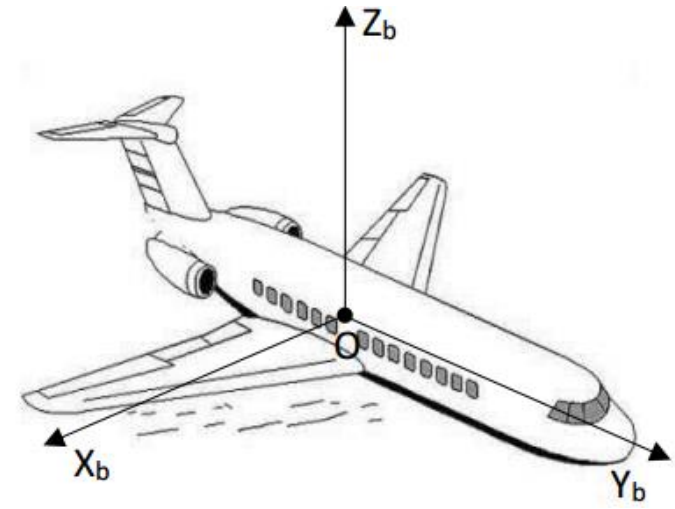

FIGURE II. CARRIER COORDINATE SYSTEM

\section{B. Attitude angle Definition}

The attitude angle of the carrier is determined by the relationship between the carrier coordinate system and the corresponding coordinate system, which is divided into Yaw angle, Pitch angle and Roll angle.

1) $\operatorname{Yaw}(\psi)$ :

The carrier rotate around $\mathrm{Z}_{\mathrm{b}}$ axis, and the angle between the projection $\mathrm{Y}_{\mathrm{b}}$ 'of the carrier $\mathrm{Y}_{\mathrm{b}}$ axis on the reference coordinate system $\mathrm{X}_{\mathrm{n}}-\mathrm{O}-\mathrm{Y}_{\mathrm{n}}$ plane and the reference coordinate system $Y_{n}$ axis is called the Yaw angle. Reference to the coordinate system $X_{n}-O-Y_{n}$ plane, clockwise rotation is positive, anticlockwise rotation is negative, range from 0 to 360 degrees.

2) $\operatorname{Pitch}(\vartheta)$ : 
The carrier rotate around $X_{b}$ axis, and the angle between the projection $\mathrm{Y}_{\mathrm{b}}$ " of the carrier $\mathrm{Y}_{\mathrm{b}}$ axis on the reference coordinate system $Z_{n}-O-Y_{n}$ plane and the reference coordinate system Yn axis is called the pitch angle. Reference to coordinate system $\mathrm{Z}_{\mathrm{n}}-\mathrm{O}-\mathrm{Y}_{\mathrm{n}}$ plane, the upward rotation is positive, the downward rotation is negative, range from -90 to 90 degrees.

\section{3) $\operatorname{Roll}(\gamma)$ :}

The carrier rotate around $Y_{b}$ axis, the angle between the projection $\mathrm{Z}_{\mathrm{b}}$ ' of the carrier $\mathrm{Z}_{\mathrm{b}}$ axis on the reference coordinate system $Z_{n}-O-X_{n}$ plane and the reference coordinate system $Z_{n}$ axis is called the roll angle. Reference to coordinate system $Z_{n}$ $\mathrm{O}-\mathrm{X}_{\mathrm{n}}$ plane, right is positive, left is negative, range from -180 $\sim 180$ degrees.

The definition of the attitude angle is shown in Figure 3:

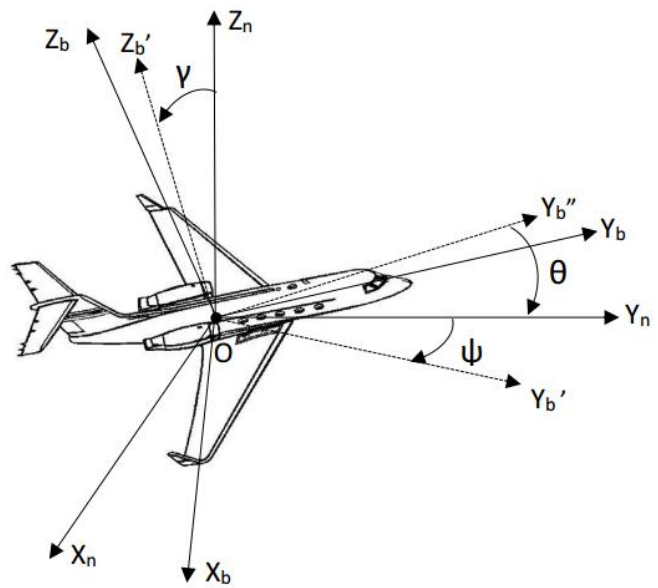

FIGURE III. THE DEFINITION OF THE ATTITUDE ANGLE

\section{Coordinate System Conversion}

After defined reference coordinate system and carrier coordinate system, the carrier coordinate systemis translated so that the origin $\mathrm{O}$ of the two coordinate systems coincide. Then the reference coordinate system is rotated so that its axis coincides with the carrier coordinate system. The angle of rotation is the attitude angle.

Any complex angular position relation of two coordinate systems can be regarded as a combination of finite basic rotation, the transformation matrix is equal to the multiplication of the basic rotation matrix. The difference in rotation order of attitude rotation matrix has different results, this paperdefined the rotation order is $\mathrm{X}->\mathrm{Y}->\mathrm{Z}$, so the three-axis basic rotation matrix is [2]:

$$
\begin{aligned}
\mathrm{C}_{\mathrm{x}}(\theta) & =\left[\begin{array}{ccc}
1 & 0 & 0 \\
0 & \cos \theta & -\sin \theta \\
0 & \sin \theta & \cos \theta
\end{array}\right] \\
\mathrm{C}_{\mathrm{y}}(\gamma) & =\left[\begin{array}{ccc}
\cos \gamma & 0 & \sin \gamma \\
0 & 1 & 0 \\
-\sin \gamma & 0 & \cos \gamma
\end{array}\right] \\
\mathrm{C}_{\mathrm{z}}(\psi) & =\left[\begin{array}{ccc}
\cos \psi & -\sin \psi & 0 \\
\sin \psi & \cos \psi & 0 \\
0 & 0 & 1
\end{array}\right]
\end{aligned}
$$

So two coordinate system rotation matrix is:

$$
=\left[\begin{array}{ccc}
\cos \gamma \cos \psi & \mathrm{C}_{\mathrm{n}} \mathrm{b}=\mathrm{C}_{\mathrm{Z}}(\psi) \mathrm{C}_{\mathrm{y}}(\gamma) \mathrm{C}_{\mathrm{X}}(\theta) & (2) \\
\sin \psi \sin \gamma \cos \psi-\sin \psi \cos \theta & \sin \theta \sin \psi+\sin \gamma \cos \theta \cos \psi \\
-\cos \gamma \cos \psi+\sin \theta \sin \gamma \sin \psi & \sin \theta \cos \psi-\sin \gamma \sin \psi \cos \theta
\end{array}\right]
$$

Where $\theta, \gamma$ and $\psi$ are the attitude angles.

\section{QUATERNION METHOD}

As the amount of calculation of the rotation matrix to calculate the attitude angle calculation is large, we use the quaternion method. Quaternion method avoids the singularity caused by Euler angle description, and can effectively combine the characteristics of sensor to compensate error. Angular velocity of gyroscope is used to update quaternion, accelerometer and magnetometer are used to compensate and calibrate quaternion.

\section{A. Calculate Attitude Angle}

Quaternion can also represent the rotation of axis, formula is $: \mathrm{R}_{\mathrm{b}}=\mathrm{QR}_{\mathrm{n}} \mathrm{Q}^{*}$, where $\mathrm{R}_{\mathrm{n}}$ is defined as a vector in the reference coordinate system, where $R_{n}=0+i x+j y+k z, Q=q 0+q 1 i$ $+q 2 j+q 3 k, q^{*}$ is the conjugate of $q$. So:

$$
\mathrm{Rb}=\left(\mathrm{q}_{0}+\mathrm{q}_{1} i+\mathrm{q}_{2 j} \mathrm{j}+\mathrm{q}_{3} \mathrm{k}\right)(0+\mathrm{ix}+\mathrm{jy}+\mathrm{kz})\left(\mathrm{q}_{0}-\mathrm{q}_{1} i-\mathrm{q}_{2 j} \mathrm{j}-\mathrm{q}_{3} \mathrm{k}\right)
$$

(3)

$$
\begin{aligned}
= & \left\{\left(\mathrm{q}^{2}+\mathrm{q}^{2}-\mathrm{q}_{2}^{2}-\mathrm{q}^{2}\right) \mathrm{x}+2\left(\mathrm{q}_{1} \mathrm{q}_{2}-\mathrm{q}_{0} \mathrm{q}_{3}\right) \mathrm{y}+2\left(\mathrm{q}_{1} \mathrm{q}_{3}+\mathrm{q}_{0} \mathrm{q}_{2}\right) \mathrm{z}\right\} \mathrm{i}+ \\
& \left\{2\left(\mathrm{q}_{1} \mathrm{q}_{2}+\mathrm{q}_{0} \mathrm{q}_{3}\right) \mathrm{x}+\left(\mathrm{q}^{2}-\mathrm{q}^{2}+\mathrm{q}^{2}-\mathrm{q}^{2}\right) \mathrm{y}+2\left(\mathrm{q}_{2} \mathrm{q}_{3}-\mathrm{q}_{0} \mathrm{q}_{1}\right) \mathrm{z}\right\} \mathrm{j}+ \\
& \left\{2\left(\mathrm{q}_{1} \mathrm{q}_{3}-\mathrm{q}_{0} \mathrm{q}_{2}\right) \mathrm{x}+2\left(\mathrm{q}_{2} \mathrm{q}_{3}+\mathrm{q}_{0} \mathrm{q}_{1}\right) \mathrm{y}+\left(\mathrm{q}^{2}-\mathrm{q}^{2}-\mathrm{q}^{2}+\mathrm{q}^{2}\right) \mathrm{z}\right\} \mathrm{k}
\end{aligned}
$$

Matrix form is:

$$
\mathrm{C}_{\mathrm{n}}^{\mathrm{b}}
$$

$$
\left[\begin{array}{ccc}
q_{0}^{2}+q_{1}{ }^{2}-q_{2}{ }^{2}-q_{3}{ }^{2} & 2\left(q_{1} q_{2}-q_{0} q_{3}\right) & 2\left(q_{1} q_{3}+q_{0} q_{2}\right) \\
2\left(q_{1} q_{2}+q_{0} q_{3}\right) & q_{0}{ }^{2}-q_{1}{ }^{2}+q_{2}{ }^{2}-q_{3}^{2} & 2\left(q_{2} q_{3}-q_{0} q_{1}\right) \\
2\left(q_{1} q_{3}-q_{0} q_{2}\right) & 2\left(q_{2} q_{3}+q_{0} q_{1}\right) & q_{0}{ }^{2}-q_{1}{ }^{2}-q_{2}{ }^{2}+q_{3}{ }^{2}
\end{array}\right]
$$

Since both $\mathrm{Cn}^{\mathrm{b}}$ and $\mathrm{Cn}^{\mathrm{b}}$ represent the rotation matrix of the reference coordinate system to the carrier coordinate system, So we can get: $\mathrm{Cn}^{\mathrm{b}}=\mathrm{Cn}^{\mathrm{b}}$, attitude angle can be obtained by comparing two formulas:

$$
\begin{gathered}
\theta=\arctan \left(\frac{2\left(q_{2} q_{3}+q_{0} q_{1}\right)}{q_{0}^{2}-q_{1}{ }^{2}-q_{2}{ }^{2}+q_{3}{ }^{2}}\right) \\
\gamma=\arcsin \left(2\left(q_{0} q_{2}-q_{1} q_{3}\right)\right) \\
\psi=\arctan \left(\frac{2\left(q_{1} q_{2}+q_{0} q_{3}\right)}{q_{0}^{2}+q_{1}^{2}-q_{2}{ }^{2}-q_{3}{ }^{2}}\right)
\end{gathered}
$$

\section{B. Update Quaternion}

The essence of quaternion update is to solve the quaternion differential equation, according to the definition of the quaternion differential equation we get the matrix as follow:

$$
\frac{\mathrm{dQ}}{\mathrm{dt}}=\left[\begin{array}{l}
q_{0}{ }^{\prime}(t) \\
q_{1}^{\prime}(t) \\
q_{2}{ }^{\prime}(t) \\
q_{3}{ }^{\prime}(t)
\end{array}\right]=\frac{1}{2}\left[\begin{array}{cccc}
0 & -w_{x} & -w_{y} & -w_{z} \\
w_{x} & 0 & w_{z} & -w_{y} \\
w_{y} & -w_{z} & 0 & w_{x} \\
w_{z} & w_{y} & -w_{x} & 0
\end{array}\right]\left[\begin{array}{l}
q_{0}(t) \\
q_{1}(t) \\
q_{2}(t) \\
q_{3}(t)
\end{array}\right]
$$


The first-order Runge-Kutta method is used to solve the differential equation, $\mathrm{Q}(\mathrm{t}+\mathrm{h})=\mathrm{Q}(\mathrm{t})+\mathrm{Tk} 1$, where $\mathrm{k} 1=\frac{\mathrm{dQ}}{\mathrm{dt}}$, $\mathrm{T}$ is the gyroscope data sampling period, $w_{x}, w_{y}$ and $w_{z}$ is the gyroscope three-axis angular velocity sampling data, and finally get the quaternion update equation is:

$$
\begin{gathered}
\mathrm{q}_{0}=\mathrm{q}_{0}+\frac{\mathrm{T}}{2}\left(-\mathrm{q}_{1} w_{x}-\mathrm{q}_{2} w_{y}-\mathrm{q}_{3} w_{z}\right) \\
\mathrm{q}_{1}=\mathrm{q}_{1}+\frac{\mathrm{T}}{2}\left(\mathrm{q}_{0} w_{x}+\mathrm{q}_{2} w_{z}-\mathrm{q}_{3} w_{y}\right) \\
\mathrm{q}_{2}=\mathrm{q}_{2}+\frac{\mathrm{T}}{2}\left(\mathrm{q}_{0} w_{y}-\mathrm{q}_{1} w_{z}+\mathrm{q}_{3} w_{x}\right) \\
\mathrm{q}_{3}=\mathrm{q}_{3}+\frac{\mathrm{T}}{2}\left(\mathrm{q}_{0} w_{z}+\mathrm{q}_{1} w_{y}-\mathrm{q}_{2} w_{x}\right)
\end{gathered}
$$

Then the quaternion is normalized:

$$
\begin{aligned}
& \text { norm }=\frac{1}{\sqrt{q_{0}{ }^{2}+q_{1}{ }^{2}+q_{2}{ }^{2}+q_{3}{ }^{2}}} \\
& \mathrm{q}_{0}=\mathrm{q}_{0} * \text { norm } \quad \mathrm{q}_{1}=\mathrm{q}_{1} * \text { norm } \quad \mathrm{q}_{2}=\mathrm{q}_{2} * \text { norm } \\
& \mathrm{q}_{3}=\mathrm{q}_{3} * \text { norm }
\end{aligned}
$$

\section{Data Fusion}

Gyroscope will be influenced by the interference torque during the flight and produce a drift error, even if the aircraft is stable, the data of gyroscope will change with time and temperature, it is unreliable for long-term use, so the data of gyroscope can not be used directly to update quaternion [3]. In order to correct these errors, this paper uses data fusion algorithm, combined with high stable data of the accelerometer and magnetometer to compensate gyroscope.

\section{1) Seeking error by accelerometer}

Get matrix $\mathrm{V}_{\mathrm{n}}$ from three elements of the third row of $\mathrm{Cn}^{\mathrm{b}}$ :

$$
\begin{aligned}
& \mathrm{Vn}=\left[\begin{array}{lll}
v_{x} & v_{y} & v_{z}
\end{array}\right] \\
& =\left[\begin{array}{ll}
2\left(q_{1} q_{3}-q_{0} q_{2}\right) & 2\left(q_{2} q_{3}+q_{0} q_{1}\right) \\
q_{3}{ }^{2}
\end{array}\right]
\end{aligned}
$$

According to the definition, these three elements are the components of three axes $\mathrm{X}_{\mathrm{b}}, \mathrm{Y}_{\mathrm{b}}, \mathrm{Z}_{\mathrm{b}}$ on carrier coordinate system. The physical meaning can be understood as the gravitational acceleration of $\mathrm{Z}$ axis on reference coordinate system turns to be the components of the three axis on carrier coordinate system.

Accelerometer measures the gravity acceleration of three axis on carrier coordinate system actually, after normalization is recorded as: $\mathrm{A}_{\mathrm{b}}=\left[\begin{array}{lll}a_{x} & a_{y} & a_{z}\end{array}\right]$. The error between $\mathrm{V}_{\mathrm{n}}$ and $\mathrm{A}_{\mathrm{b}}$ is the error between the gyro attitude estimate and the actual measured attitude of the accelerometer, and the error is expressed in the form of vector cross product [4]:

$$
\mathrm{Ea}=\left[\begin{array}{l}
e_{a x} \\
e_{a y} \\
e_{a z}
\end{array}\right]=\mathrm{Vn} \times \mathrm{Ab}=\left[\begin{array}{l}
v_{y} a_{z}-v_{z} a_{y} \\
v_{z} a_{x}-v_{x} a_{z} \\
v_{x} a_{y}-v_{y} a_{x}
\end{array}\right]
$$

\section{2) Seeking error by magnetometer}

When aircraft is in the horizontal state, the accelerometer can not measure the rotation around the $\mathrm{Zb}$ axis: Yaw angle $(\psi)$, so this paper consider the integration of magnetometer data on the basis of the integration of accelerometer for gyroscope error compensation [5].

The normalized data of the magnetometer on carrier coordinate system are: $\mathrm{m}_{\mathrm{b}}=\left[\begin{array}{lll}m_{x} & m_{y} & m_{z}\end{array}\right]$. At the same time, the rotation matrix of carrier coordinate systemto the reference coordinate systemis obtained from the matrix $\mathrm{Cn}^{\mathrm{b}}$ as follows:

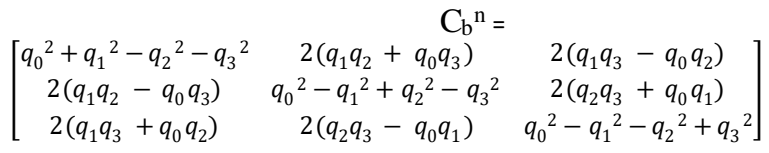

Converts the magnetometer data $\mathrm{m}_{\mathrm{b}}$ on carrier coordinate system into the reference coordinate system to obtain the converted data:

$$
\mathrm{M}_{\mathrm{n}}=\left[\begin{array}{lll}
M_{x} & M_{y} & M_{z}
\end{array}\right]=\mathrm{m}_{\mathrm{b}} * \mathrm{C}_{\mathrm{b}}^{\mathrm{n}}
$$
$\left.q_{0} q_{2}\right) m_{2}$

$\mathrm{M}_{\mathrm{x}}=\left({q_{0}}^{2}+{q_{1}}^{2}-{q_{2}}^{2}-{q_{3}}^{2}\right) m_{x}+2\left(q_{1} q_{2}-q_{0} q_{3}\right) m_{y}+2\left(q_{1} q_{3}+\right.$

$\mathrm{M}_{\mathrm{y}}=2\left(q_{1} q_{2}+q_{0} q_{3}\right) m_{x}+\left(q_{0}^{2}-q_{1}{ }^{2}+q_{2}{ }^{2}-q_{3}{ }^{2}\right) m_{y}+2\left(q_{2} q_{3}-\right.$ $\left.q_{0} q_{1}\right) m_{z}$ $\left.q_{3}{ }^{2}\right) m_{z}$

$\mathrm{M}_{\mathrm{z}}=2\left(q_{1} q_{3}-q_{0} q_{2}\right) m_{x}+2\left(q_{2} q_{3}+q_{0} q_{1}\right) m_{y}+\left(q_{0}{ }^{2}-q_{1}{ }^{2}-q_{2}{ }^{2}+\right.$

In the ideal state, the $Y_{n}$ of reference coordinate systemaxis points to the north, so the magnetometer component of $X_{n}$ axis is 0 , according to the actual estimated magnetometer data $M_{n}$ on reference coordinate system, we can calibrated magnetometer data $\mathrm{M}_{\mathrm{n}}{ }^{\prime}$ of three axis on reference coordinate system in ideal state:

$$
\mathrm{M}_{\mathrm{n}}{ }^{\prime}=\left[\begin{array}{lll}
0 & M_{y}{ }^{\prime} & M_{z}^{\prime}
\end{array}\right]=\left[\begin{array}{lll}
0 & \sqrt{M_{x}^{2}+M_{y}^{2}} & M_{z}
\end{array}\right]
$$

Then, magnetometer data $\mathrm{M}_{\mathrm{n}}{ }^{\prime}$ on reference coordinate system in the ideal state is converted to the carrier coordinate system by the rotation matrix $\mathrm{Cn}^{\mathrm{b}}$, and magnetometer data on carrier coordinate systemin the ideal state is obtained:

$$
\mathrm{m}_{\mathrm{b}}{ }^{\prime}=\left[\begin{array}{lll}
m_{x}{ }^{\prime} & m_{y}{ }^{\prime} & m_{z}{ }^{\prime}
\end{array}\right]
$$

where:

$$
\begin{aligned}
& \mathrm{m}_{\mathrm{x}}{ }^{\prime}=2\left(q_{1} q_{2}+q_{0} q_{3}\right) M_{y}{ }^{\prime}+2\left(q_{1} q_{3}-q_{0} q_{2}\right) M_{z}{ }^{\prime} \\
& \mathrm{m}_{\mathrm{y}^{\prime}}=2\left(q_{0}{ }^{2}-q_{1}{ }^{2}+{q_{2}}^{2}-{q_{3}}^{2}\right) M_{y}{ }^{\prime}+2\left(q_{2} q_{3}+q_{0} q_{1}\right) M_{z}{ }^{\prime} \\
& \mathrm{m}_{\mathrm{z}}{ }^{\prime}=2\left(q_{2} q_{3}-q_{0} q_{1}\right) M_{y}{ }^{\prime}+\left({q_{0}}^{2}-q_{1}{ }^{2}-q_{2}{ }^{2}+q_{3}{ }^{2}\right) M_{z}{ }^{\prime}
\end{aligned}
$$

In the ideal state, the error between the magnetometer data $\mathrm{mb}_{\mathrm{b}}$ ' and the actual measured magnetometer data $\mathrm{mb}$ is expressed in the form of vector cross product: 


$$
\mathrm{E}_{\mathrm{m}}=\left[\begin{array}{c}
e_{m x} \\
e_{m y} \\
e_{m z}
\end{array}\right]=\mathrm{m}_{\mathrm{b}}, \times \mathrm{m}_{\mathrm{b}}=\left[\begin{array}{l}
m_{y}{ }^{\prime} m_{z}-m_{z}{ }^{\prime} m_{y} \\
m_{z}{ }^{\prime} m_{x}-m_{x}{ }^{\prime} m_{z} \\
m_{x}{ }^{\prime} m_{y}-m_{y}{ }^{\prime} m_{x}
\end{array}\right]
$$

So combined with the error $\mathrm{E}_{\mathrm{a}}$ obtained by accelerometer, the actual error is:

$$
\mathrm{E}=\mathrm{E}_{\mathrm{a}}+\mathrm{E}_{\mathrm{m}}=\left[\begin{array}{c}
e_{x} \\
e_{y} \\
e_{z}
\end{array}\right]=\left[\begin{array}{c}
v_{y} a_{z}-v_{z} a_{y}+m_{y}{ }^{\prime} m_{z}-m_{z}{ }^{\prime} m_{y} \\
v_{z} a_{x}-v_{x} a_{z}+m_{z}{ }^{\prime} m_{x}-m_{x}{ }^{\prime} m_{z} \\
v_{x} a_{y}-v_{y} a_{x}+m_{x}{ }^{\prime} m_{y}-m_{y}{ }^{\prime} m_{x}
\end{array}\right]
$$

\section{3) 4.3.3 Data Fusion and Attitude Angle}

According to the obtained error term $\mathrm{E}$, the controller will continue to function as long as the error exists, by using the PI controller to filter and fuse it into the data of gyroscope. The fusion data integral term is:

$$
\begin{aligned}
& e_{x} \text { Int }=e_{x} \operatorname{Int}+K i^{*} e_{x} \\
& e_{y} \operatorname{Int}=e_{y} \operatorname{Int}+K i^{*} e_{y} \\
& e_{z} I n t=e_{z} I n t+K i^{*} e_{z}
\end{aligned}
$$

Gyroscope data after fusion is:

$$
\begin{gathered}
w_{x}=w_{x}+\mathrm{Kp}^{*} \mathrm{e}_{\mathrm{x}}+\mathrm{e}_{\mathrm{x}} \text { Int } \\
w_{y}=w_{y}+\mathrm{Kp} * \mathrm{e}_{\mathrm{y}}+\mathrm{e}_{\mathrm{y}} \text { Int } \\
w_{z}=w_{z}+\mathrm{Kp}^{*} \mathrm{e}_{\mathrm{z}}+\mathrm{e}_{\mathrm{z}} \text { Int }
\end{gathered}
$$

The effect of error cancellation depends on the proportional control parameter $\mathrm{Kp}$ and the integral control term parameter Ki. After compensating the gyro data, we can use the equation (7) to obtain the attitude quaternion, and finally use the formula (5) to obtain the aircraft attitude angle.

\section{EXPERIMENT AL RESULTS}

The experiment mainly uses the MCU's UART interface to communicate with the host computer, transmitted the calculated attitude angle data to the host computer in real time, and produce the corresponding waveform. The attitude angle waveform shown in Figure 4:

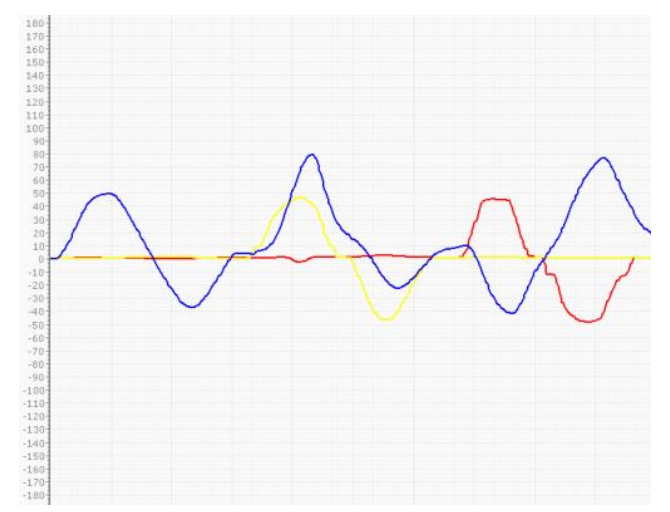

FIGURE IV. ATT ITUDE ANGLE WAVE GRAPH

where the vertical axis for angle, range from $-180^{\circ}$ to $180^{\circ}$; The blue waveform represents Yaw angle; the red waveform represents Roll angle and the yellow waveform represents pitch angle. After compared with waveform data and actual angle, indicating that the algorithm has high accuracy, small error, fast and is able to complete the task of calculating attitude during the aircraft actual flight.

\section{CONCLUSION}

In this paper, we used quaternion algorithm, and according to the characteristics of accelerometer and magnetometer data, they are fused to gyroscope data, which effectively eliminates the error. The effectiveness of the algorithm is verified by experiments, and it has great application prospect in the attitude calculation of the aircraft.

\section{REFERENCES}

[1] Chang Guoquan, Dai Guoqiang. Design of Flight Control System Based on STM32 for Four-axis aircraft[J]. Microcontrollers \& Embedded Systems,2015(2),29-32.

[2] Qin Yongyuan. Inertial navigation [M].Beijing:Science Press, 2006,287304.

[3] Hu Hengzhang. Gyroscope Drift Test Principle and Its Experimental Technology [M].Beijing:National Defense Industry Press, 1981,2-22.

[4] Guan Yuewei, He Boxian, Yu Renqing, Ding Guangwe. Realization of Attitude Angle Algorithm for Gyroscope Based on Quaternion [J].Computer programming skills and maintenance,2015(9),32-34.

[5] Dai Lei, Qi Juntong, Wu Chong, Han Jianda. Error Analysis and Calibration of Magnetic Compass of Rotorcraft [J].Robot,2012(34),418423. 